\title{
A Study on Development of an Integrated IoT Service Platform Using Spatial Information
}

\author{
Yun-Gil Lee1)
}

\begin{abstract}
This research aimed to develop an advanced IoT service platform based on spatial information. This technology is similar to the existing Internet of Things (IoT) platform in that it manages IoT devices in an integrated manner. However, it differs from the existing IoT platform in that it uses spatial information to recognize user situation and adapt the service accordingly. This ability to understand the user's situation based on location can enable more advanced IoT services. Through this study, the possibilities of advanced IoT services using spatial information were tested. To establish a service platform, traditional spatial information modeling techniques as a location model for IoT service platform was used. To evaluate the performance of the developed service platform, a testbed was constructed. In addition, the device and system configuration for location tracking was configured using Beacons, Arduino, and mobile devices. An input/output module and a location information monitoring system were developed and used for smooth testbed operation. Through the operation of the pilot, we confirmed that spatial information can be integrated into IoT service system, and IoT service platform based on spatial information can provide more detailed user services.

Keywords: Internet of Things, Spatial Information, Location Based Service, Service Platform, User Experience
\end{abstract}

\section{Introduction}

As the dissemination and utilization of IoT technologies is gradually increasing, the demand for more advanced IoT services is also increasing. Research on the development of more advanced IoT services is actively being conducted, particularly with respect to IoT services that can utilize spatial information. This is because all IoT devices are placed in a physical architectural space, and occupants of IoT services also operate in space[1][2]. Spatial information is not only physical information but also contextual information associated with a user's location; understanding this information may enable a more appropriate IoT service. This study aimed to develop an IoT Internet platform based on spatial information. This technology is similar to the

Received(April 19, 2020), Review Result(1st: June 4, 2020, 2nd: July 23, 2020), Accepted(August 28, 2020)

1) (Associate Professor) 31499 Dept. of Architecture, Hoseo Univ., Baebang-eup, Asan-si, Chungcheongnam-do, Republic of Korea

email: yglee@hoseo.edu 
existing IoT platform in that it manages and services IoT devices in the space but is different in its ability to recognize and adapt to the user's situation using spatial information. In this study, we explored the possibility of advanced IoT services using spatial information[3][4]. [Fig. 1] shows the idea of this study which is a kind of the integrated service platform using spatial information.

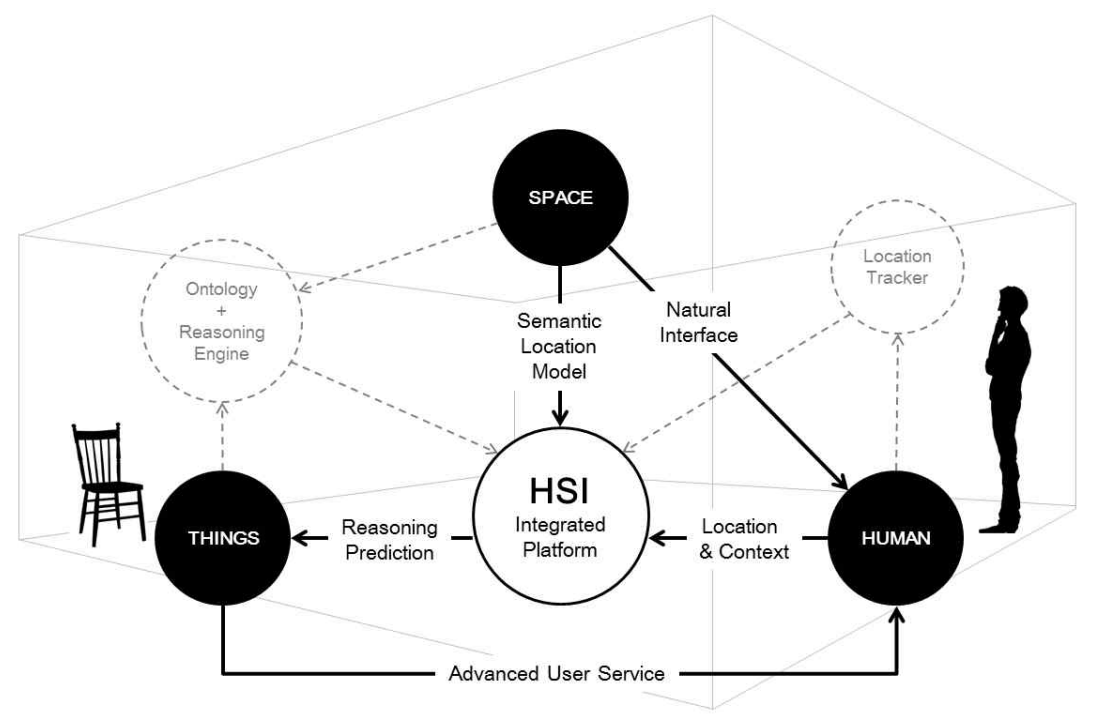

[Fig. 1] Conceptual Diagram of Spatial Information-based IoT Service[5]

\section{IOT and Spatial Information}

The Internet of Things is a concept that shares the Mac with Machine to Machine (M2M) technology or Ubiquitous, which has been studied extensively. However, the difference between these and IoT services can be said to construct a more intelligent and integrated service environment[6]. Recent advancements in IoT technologies aim to build an active environment in which humans, objects, and services perform sensing, networking, and information processing in a cooperative manner. However, the realization of the Internet of Things in earnest must go beyond simple connection and one-dimensional interaction and be connected to the placeness mediated by indoor spatial information; it is essential to recognize the context associated with the place in order to provide advanced services. An IoT service that does not take into account context information associated with a place is only a simple M2M technology[7][8].

In his paper, Jang $\mathrm{Mi} \mathrm{Na} \mathrm{Ra}(3,2016)$ proposed an IoT service that recognizes spatial information as a context for user services with the concept of Human Space Interaction (HSI). 
This provides a location-based service to the user by using spatial information to generate a kind of location map. Generally, more advanced IoT services can be provided by using spatial information as an integrated platform (As an Integrated Platform), as a natural interface (As a Natural Interface), and as context-aware location information (As a Semantic Location Map) [5][9].

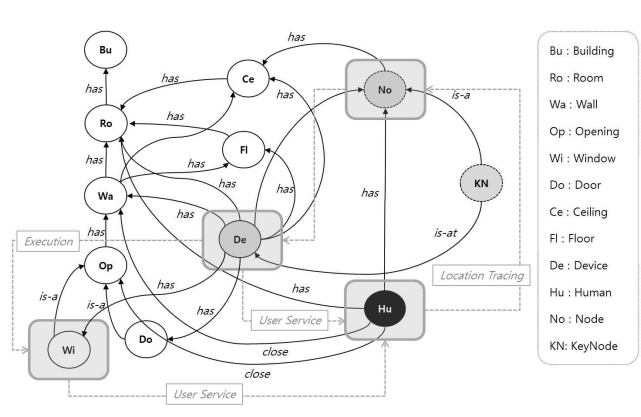

[Fig. 2] Ontology Data Model for Spatial Information based IoT Service

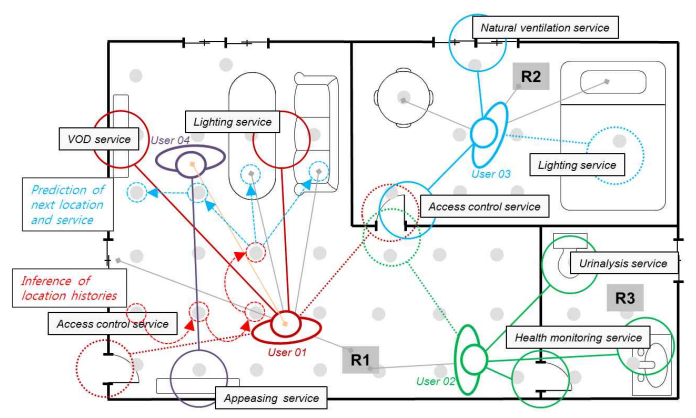

[Fig. 3] Various User Serviceability Considering Spatial Situation

\section{Development of IOT Platform Using Spatial Information}

\subsection{Designing of IOT Platform based on Spatial Information}

This study explored the possibilities of advanced IoT services using spatial information and showed how IoT platforms based on spatial information can be constructed and developed. Specifically, the IoT platform to actively utilize spatial information to recognize and integrate the user's location was designed. To this end, it was necessary to create a cognitive information system that recognized spatial information, to develop a technology that inferred the situation, and to configure a service environment that recognized the situation according to the user's location and provided appropriate services. In order to create a cognitive information system that recognizes spatial information, information modeling was performed using sensors, devices, and services based on traditional physical space modeling techniques. [Fig. 2] shows the modeling of a situation-aware information system based on spatial information for IoT platform development. It is an ontology system that objectizes the elements that make up the physical space and defines their relations. In addition, the relationship between the devices that provide location, service, and physical space elements is defined, and the flow of information is plotted. While various technical methods for recognizing the user's location exist, this study 
used a method based on the location-tracking method Beacon[10].

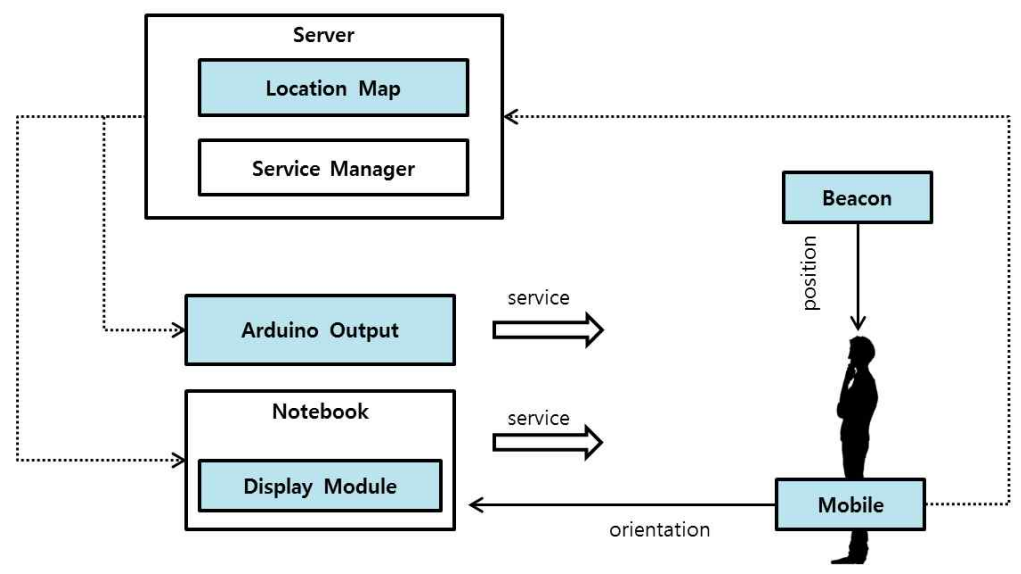

[Fig. 4] Conceptual Diagram of System Configuration for Location Tracking

[Fig. 3] shows how modeled spatial information can be used to apply to services[11]. The user's location is improved by the triangulation technique using the numerical location information provided by each node, and the user's location is inferred through spatial information and comparison operations. When a location in the user's space is derived, possible services are searched and prepared based on the user's location. Here, the KeyNode is designed to reduce the burden of computation and of device installation for location tracking and service provision, and to provide more appropriate services. [Fig. 4] shows how the system for location tracking is constructed. As described above, location tracking based on Beacon was used, and it worked by acquiring physical information from a user's mobile device calculating it on a server. The configured spatial information (Location Map) is stored on the server side to infer the user's situation in real time. The server derives an appropriate service according to the user's location and requests the service device.

\subsection{Development of a Spatial Information-Based IoT Platform Prototype}

The researchers developed a platform prototype that integrates spatial information to provide IoT services. A Windows PC was used as a server, and a Raspberry Pi 3 was used and a display device as an IoT client. An Android-based smartphone was used to obtain the user's location information from the installed Beacon, and the user's orientation was extracted through the built-in direction sensor. The server development was written in the Windows-based net framework C\#, and the client was developed in Python 3 on Raspbian OS. Like Arduino, the 
platform was developed as an open source IoT service and calculates possible services based on spatial information, user location, and gaze mounted on the server. [Fig. 5] shows the physical and logical system configuration of the IoT platform[12-14].

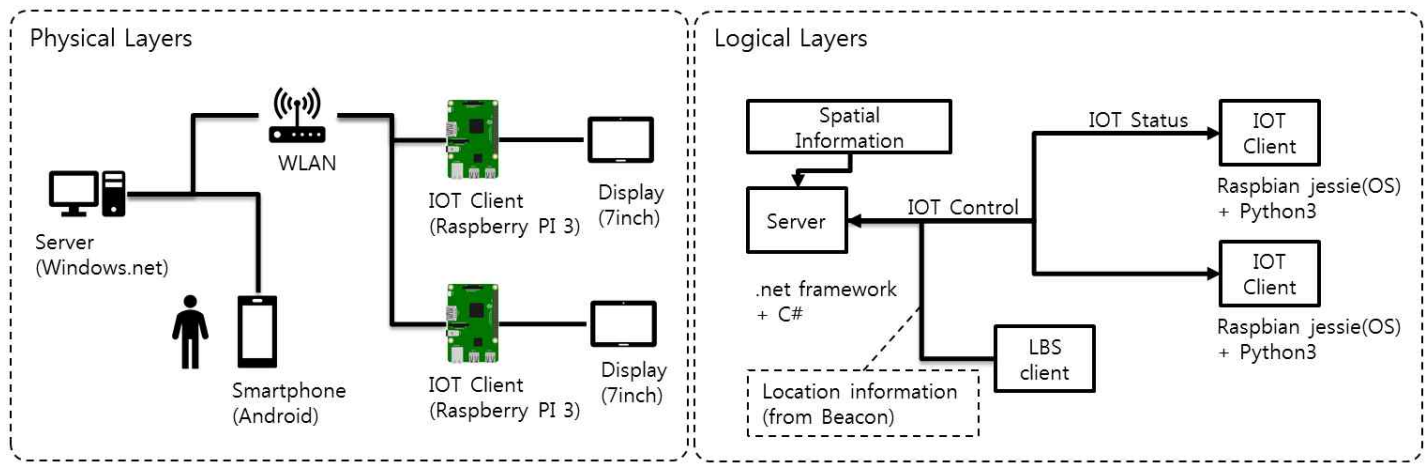

[Fig. 5] Physical and Logical System Configuration of IoT Platform based on Spatial Information

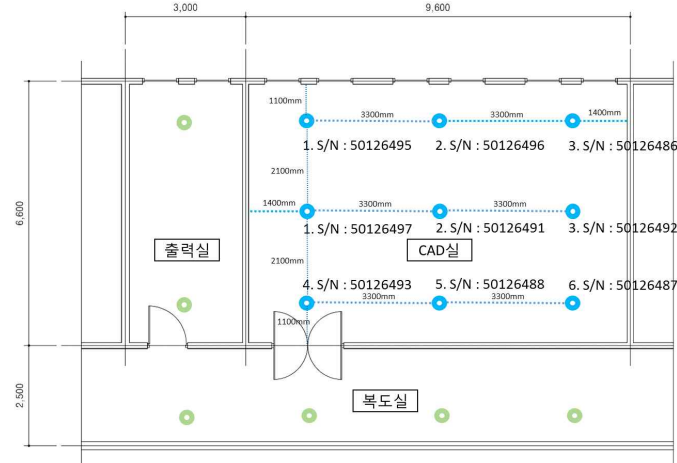

[Fig. 6] Deployment of Beacons to Build Testbed

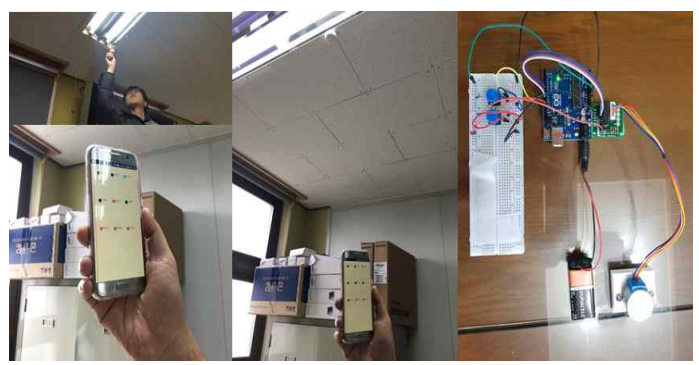

[Fig. 7] Acquisition of Location / Direction Information through Mobile Device and Input /

Output of Arduino

\subsection{Construction of Device Facilities and Contents for Pilot Service}

A testbed for a pilot service based on our IoT platform was built. Location and direction information were obtained through Beacon and through mobile devices, and a user's location in the space was calculated through a triangulation algorithm. I/O devices were developed using Arduino modules to build the testbed. [Fig. 6] shows how the beacons were placed in the space to build the testbed. [Fig. 7] shows the configuration of Arduino and mobile devices to acquire location and direction information through mobile devices. In addition, a video (image) input/output and editing module was developed to facilitate the construction of Arduino and 
IoT services as shown in [Fig. 8]. This was done so that the system could be used for various purposes (education, experiment, etc.) in the future. Through the module in [Fig. 8], efforts to develop for input/output can be minimized.

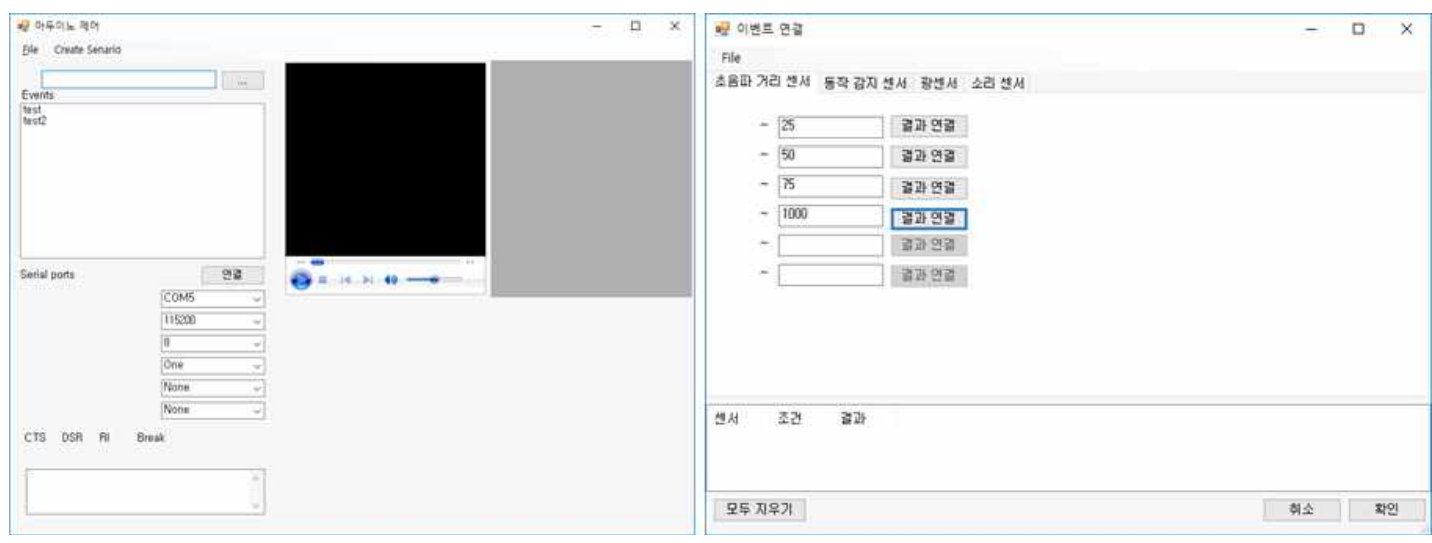

[Fig. 8] Video (image) Input / Output and Editing Module for Easy-setting of Arduino and IOT Service
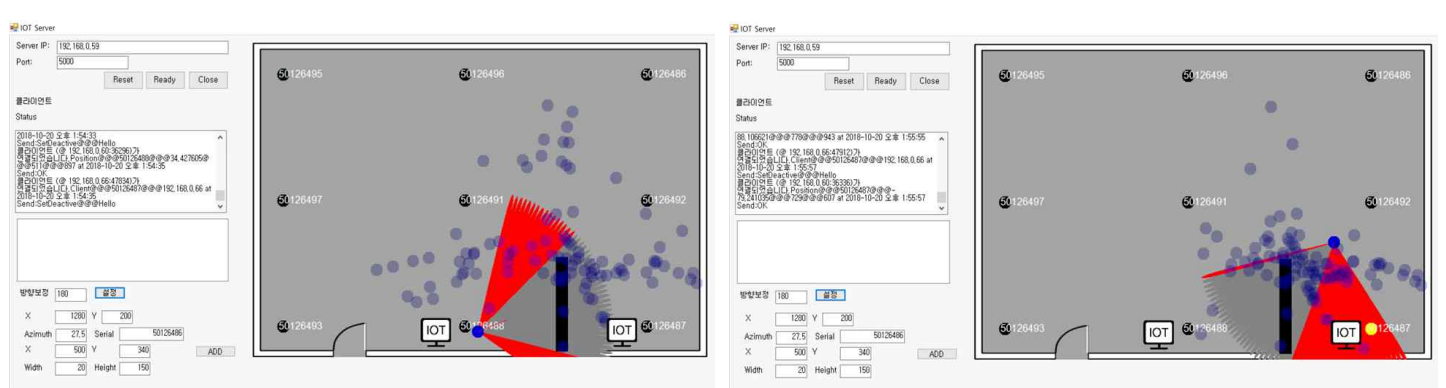

[Fig. 9] Monitoring System for User Location and IOT Service in Space

\subsection{Pilot Operation of Spatial Information-based Internet of Things Service}

The internet-based IoT service was pilot-operated, and the monitoring system was developed and operated as follows to more closely and visually check the operation status of the system shown in [Fig. 9]. [Fig. 10] shows the execution of an IoT service platform based on spatial information that provides different services depending on the user's location in a testbed environment. IoT service was provided considering the location and obstacles to the user. In other words, as shown in [Fig. 10], the platform can be managed so that possible IoT services can be classified according to the situation of the space and the location and direction of the 

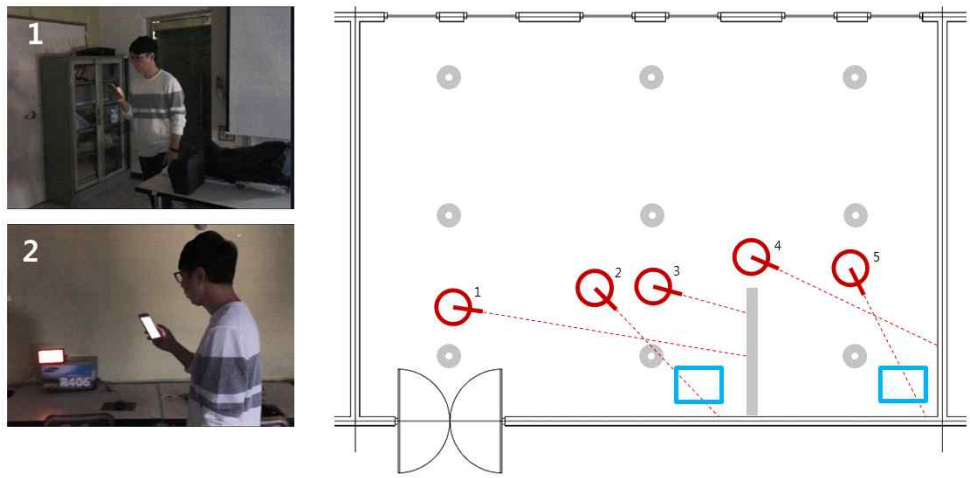
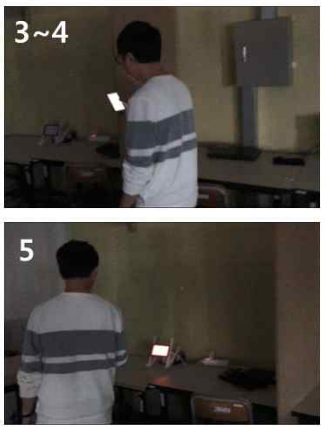

[Fig. 10] User Evaluation of Developed IOT Platform based on Spatial Information

\section{Conclusion}

This study aimed to develop an internet-based IoT platform based on spatial information, and explained the development of a prototype of the said platform. Through the operation of the pilot, the researchers confirmed that spatial information can be integrated into IoT service system, and IoT service platform based on spatial information can provide more detailed user services. In the future, it will be necessary to build more diverse service situations to improve the performance of our system.

\section{Acknowledgement}

This work was supported by the National Research Foundation of Korea (NRF) grant funded by the Korea government (MSIT) (NRF-2018R1A2B6005827).

\section{References}

[1] J. Gubbia, R. Buyyab, S. Marusica, M. Palaniswamia, Internet of Things (IoT): A vision, architectural elements, and future directions, Future Generation Computer Systems, (2013), Vol.29, No.7, pp.1645-1660.

[2] X. Wang, J. Shang, F. Yu, J. Yan, Indoor Semantic Location Models for Location-Based Services, International Journal of Smart Home, (2013), Vol.7, No.4, pp.127-136.

[3] J. A. Stankovic, I. Lee, A. Mok, R. Rajkumar: Opportunities and obligations for physical computing 
systems, Computer, (2005), Vol.38, No.11, pp.23-31.

[4] M. A. Feki, F. Kawsar, M. Boussard, L. Trappeniers, The internet of things: the next technological revolution, Computer, (2013), Vol.46, No.2, pp.24-25.

[5] Mi Na Ra Jang, Chan Yang Suhr, Yun Gil Lee, Developing a the Advanced IoT (Internet of Things) Technology Based on Spatial Information, Communications in Computer and Information Science, (2016), Vol.618, No.1, pp.416-419.

[6] J. Höller, V. Tsiatsis, C. Mulligan, S. Karnouskos, S. Avesand,D. Boyle, From Machine-to-Machine to the Internet of Things: Introduction to a New Age of Intelligence, Netherlands: Elsevier, (2014)

[7] J. H. Yu, Y. G. Lee, A Basic Study on Utilization of Building Information for Internet of Things (IoT) Simulation System Development, Asia-pacific Journal of Multimedia Services Convergent with Art, Humanities, and Sociology, (2018), Vol.8, No.1, pp.867-874.

[8] S. Kaur, A. Hans, N. Singh, An Overview to Internet of Things (IOT), International Journal of Future Generation Communication and Networking, (2016), Vol.9, No.9, pp.239-246.

[9] C. Jiantao, Z. Xiaojun, Study of IoT Terminal Interface Platform Based on Embedded Technology and Zigbee Protocol, International Journal of Future Generation Communication and Networking, (2016), Vol.9, No.6, pp.55-64.

[10] https://kontakt.io/beacon-basics/what-is-a-beacon/, Apr 16 (2020).

[11] H. J. Sim, Y. G. Lee, Developing an Internet of Things (IoT) Service System Based on Spatial Context, Communications in Computer and Information Science, (2018), Vol.852, No.3, pp.510-514.

[12] https://www.raspberrypi.org/, Apr 16 (2020)

[13] https://kontakt.io/beacon-basics/what-is-a-beacon/, Apr 16 (2020)

[14] https://www.arduino.cc/, Apr 16 (2020)

[15] D. Ko, J. Choi, Forest Lodge Room Navigation Algorithm Based on Beacon, International Journal of Grid and Distributed Computing, (2017), Vol.10, No.12, pp.1-10.

[16] J. Jin, M. Lee, Building Customizable Beacon Services for Pattern-specifiable Beacons, International Journal of Grid and Distributed Computing, (2018), Vol.11, No.4, pp.49-58. 